\title{
Creating a Taxonomy of Mathematical Errors for Undergraduate Mathematics
}

Shauna Ford, School of Mathematics, Cardiff University, UK. Email: FordS6@cardiff.ac.uk. Jonathan Gillard, School of Mathematics, Cardiff University, UK. Email: GillardJW@cardiff.ac.uk. Mathew Pugh, School of Mathematics, Cardiff University, UK. Email: PughMJ@cardiff.ac.uk.

\begin{abstract}
In this paper we develop a taxonomy of errors which undergraduate mathematics students may make when tackling mathematical problems. We believe that a taxonomy would be useful for staff in giving feedback to students, and would facilitate students' higher-level understanding of the types of errors that they could make.
\end{abstract}

Keywords: assessment, feedback, errors.

\section{Introduction}

There has been a considerable amount of research over the last century into mathematical errors (Radatz, 1980). Typically this research is in the context of learning mathematics in school (e.g. Radatz, 1980; Matz, 1982; Kieran, et al., 1990; Foster, 2007). Such studies tend to focus therefore on errors which are either arithmetic or algebraic in nature, such as errors in long division, or misinterpreting $(a+b)^{2}$ as $a^{2}+b^{2}$. An approach which seems to receive particular attention in the U.S. is error analysis (e.g. Ashlock, 2010; Idris, 2011). In this approach pupils' errors are systematically recorded by the teacher, and analysed for patterns so that teacher can then plan what potential remedial action will be necessary to correct any underlying misconceptions.

Whilst research into errors made by pupils in a school context can be of benefit to teachers in higher education, the contexts are also very different. School pupils will possess a wide range of mathematical abilities, and many will have a dislike or even fear of mathematics. On the other hand mathematics undergraduate students possess a strong mathematical ability and have chosen to study the subject further. Thus one would hope that many of the errors made by pupils, resulting from a misunderstanding of even basic concepts within mathematics, would not be made by mathematics undergraduates. The approach to mathematics also tends to be very different in the two contexts. In school the focus is almost exclusively on algorithms to solve problems, whilst undergraduate mathematics will also focus on understanding concepts and proving results. Therefore the types of error made in higher education will typically be of a different nature to those in primary or secondary education.

Much of the above research, as well as more general studies on mathematical errors, focuses on understanding the underlying cognitive causes of these errors, either in order to understand the cause of specific errors, or more generally to identify the mechanisms underlying these errors. It is argued that most mathematical errors are causally determined, and very often systematic (Radatz, 1980). Radatz (1979) identified five error categories: (1) errors due to language difficulties, (2) errors due to difficulties in obtaining spatial information, (3) errors due to deficient mastery of prerequisite skills, facts, and concepts, (4) errors due to incorrect associations or rigidity of thinking, and (5) errors due to the application of irrelevant rules or strategies. Ben-Zeev (1998) constructed a taxonomy of mathematical errors and attempted to identify the causes of these errors by integrating findings from different studies. The focus in this and other research is to understand why a student makes an error. For example, a student may over-generalize an algorithm which holds in one context to a structurally 
similar context where the algorithm no longer works, something Ben-Zeev calls syntactic induction (Ben-Zeev, 1998).

It will often however be difficult, if not impossible, to diagnose the underlying error in a student's reasoning or understanding solely from the student's written solution to a problem. Therefore this paper will not focus on this, but rather on classifying the particular types of errors students make when attempting to solve mathematical problems. Such a classification should provide enough details so that a student can identify what it is they have done wrong, whilst keeping the number of classes as small as possible. We believe that creating a taxonomy of errors is useful for the following reasons:

- it will be a useful resource for students to see which errors to avoid, some of which may not have been appreciated previously;

- it could be incorporated into a feedback tool for lecturers to enrich the feedback offered to students;

- it would allow for the consideration of relationships between different types of error.

Whilst this paper implicitly assumes that the students we consider are undergraduate mathematics students, the developments in this paper could also be applied to GCSE or A-level mathematics students.

This work was undertaken as part of an undergraduate summer project by the first author. The paper is structured as follows. In section 2 we consider the definition of error that we use in this paper, and what causes errors to take place, before relating this information to errors in mathematics. The taxonomy is given in section 3 .

\section{Human Error}

Human error is a failure of a planned action to achieve a desired outcome. Errors can be made in one of two ways - either the plan itself may be inadequate, or else the execution of that plan may include actions that are unintentional and which do not lead to the desired outcome, as illustrated in figure 1.

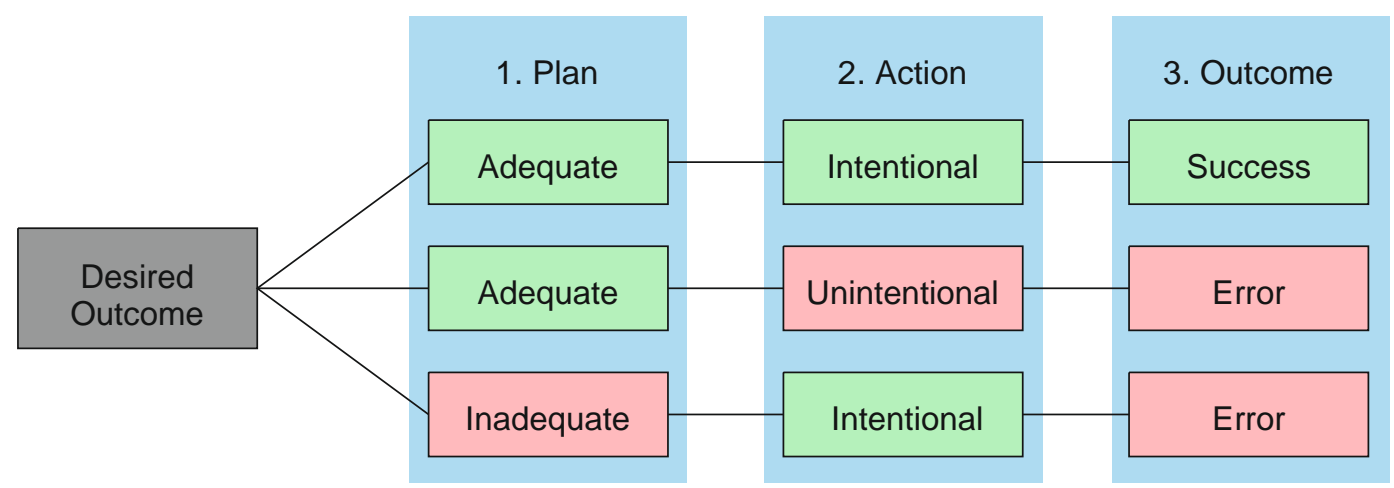

Figure 2. Occurrence of Human Errors.

Failures in planning are often referred to as mistakes rather than errors. There are two types of mistakes: knowledge-based and rule-based (Reason, 1990). Knowledge-based mistakes occur when an individual has an inability to reach an end goal because of a lack of knowledge. Rule-based mistakes occur when an individual wrongly modifies an established process. Such mistakes are more 
likely to go unnoticed when the outcome is not specifically known. The modification is likely to be informed by previous successful experiences (Rasmussen, 1986, p.102). Rule-based mistakes fall into two categories:

- Misapplication of a good rule: Occurs when an individual applies a rule which may be perfectly adequate in another situation, but which may not meet the conditions and demands of the problem being considered (Reason, 1990, p.75). Such errors are more likely to occur when an individual has applied the rule successfully for a previous problem.

- Application of a bad rule: A good rule may become bad following changes that an individual makes that are not thoroughly considered. This may be from the alterations not being managed appropriately, or the creation of a bad rule from incorrect knowledge. This can appear on varying levels; the rule could be entirely wrong, the rule may be clumsy or inefficient but still achieve the desired outcome, or the rule could be inadvisable since whilst leading to a good approximate solution, repeated use may worsen this approximation.

Unintentional actions are classed as skill-based errors. These often occur when implementing elementary or standard procedures, due to a lack of consciousness or control (Rasmussen, 1986, p.100). Skill-based errors fall into two categories: memory lapses and slips of actions.

- Memory lapse: These errors include losing place in a sequence of steps, forgetting to do something, or forgetting the overall plan entirely.

- Slip of action: An unintentional action that occurs at the point of execution. This error is often caused by a process being performed subconsciously, skipping or reordering steps in a procedure, or experiencing a distraction.

The skill-rule-knowledge framework described above only offers a partial account of possible deviant behaviour (Reason, 1990). Humans plan and execute their actions in social environments that may affect their performance. Whilst mistakes and skill based errors are defined as errors made in the individual's cognitive stages, their behaviour may also be altered by the situation's social context. Violations are deliberate alterations considered necessary by the individual to adjust to external influences (Reason, 1990, p.195). Hence the violator is not always entirely blameworthy for the decision made. The following three types of violation are distinguished:

- Routine violations: These occur due to natural instinct to take the process that requires the least amount of effort. This becomes habitual and forms a set pattern of errors in their behaviour.

- Situational violations: An individual alters their behaviour due to a change in their social surroundings. These changes can include excessive time pressure, stress, workplace design, and inadequate or inappropriate equipment.

- Exceptional violations: These occur when an individual adopts a cause of action known to be usually incorrect but determines that the current situation is an exception.

Violations and errors from the previous skill-rule-knowledge framework can coincide or appear alone. The classification of human errors described above is illustrated in figure 2. 


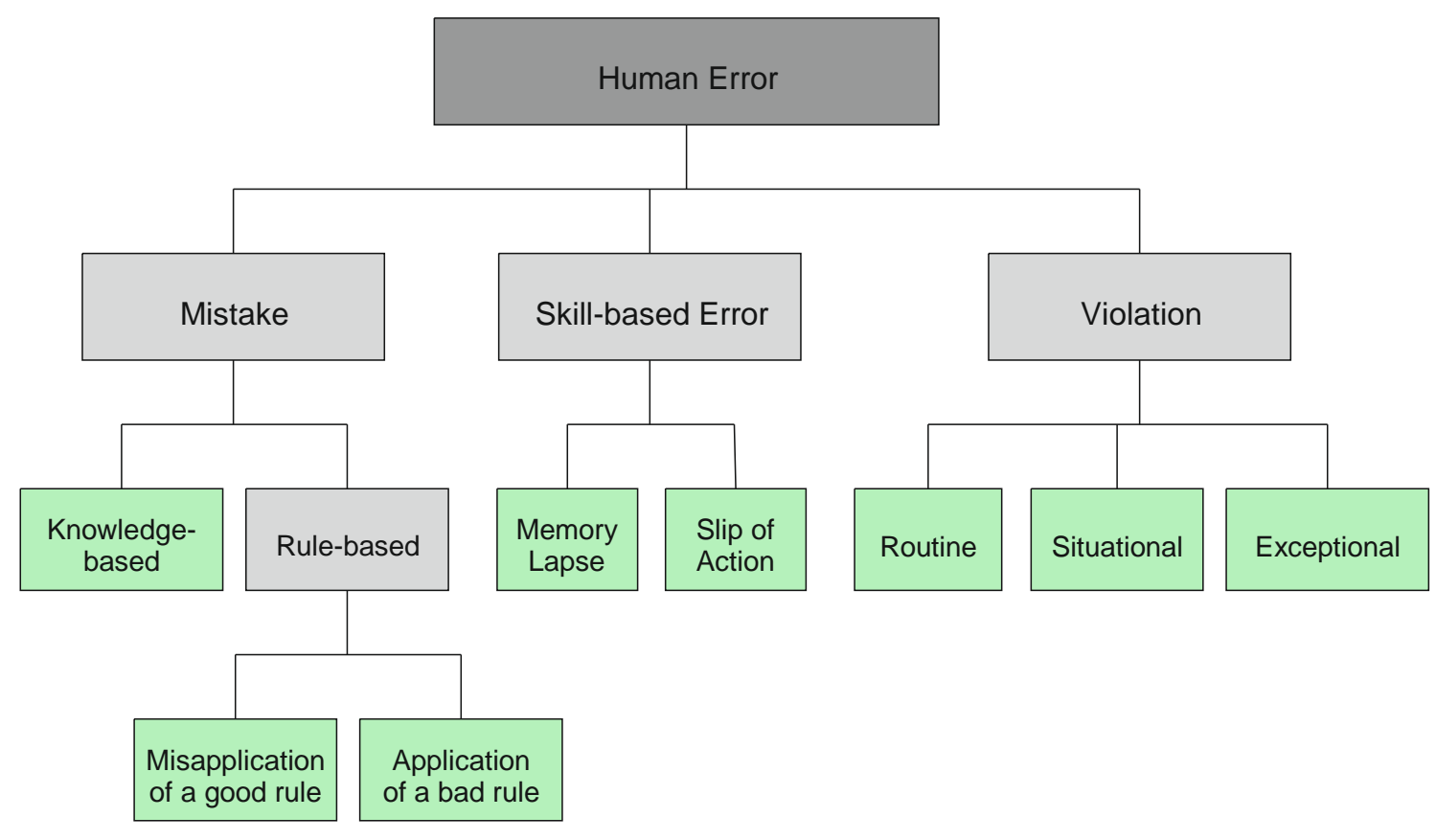

Figure 2. Human Error Types.

How might these types of errors appear in the context of a student attempting a mathematics problem? Suppose a student was answering the question:

Differentiate the function $f(x)=3 x \sin x^{2}$.

This requires using the product and chain rules to obtain an answer of $6 x^{2} \cos x^{2}+3 \sin x^{2}$. A 'slip of action' might be manifested as a numerical slip-up (such as writing the coefficient of the derivative as 5 rather than 6 , possibly through subconsciously confusing $2 \times 3=6$ with $2+3=5$ ), or a careless error in writing the solution (such as writing $\cos x$ by mistake). If the student could not recall the chain rule then this would be a 'memory lapse', whereas if they did not know the chain rule then they would likely make a 'knowledge-based' error. If they had incorrectly recalled the chain rule, then the error would be an 'application of a bad rule'. On the other hand, if the student had (wrongly) integrated the function correctly, they are likely to be guilty of 'misapplication of a good rule'. A 'routine violation' could occur if a student had made the same error often enough so that they no longer realised it was an error, for example, writing $-\cos x$ for the derivative of $\sin x$. A 'situational violation' might be more likely if the student had to answer this question in an examination, perhaps due to the stress and time-pressures of the situation. An 'exceptional violation' may occur if a student is presented with the question 'Show that the derivative of $f(x)=3 x \sin x^{2}$ is given by $f^{\prime}(x)=6 x^{2} \cos x^{2}+3 \sin x^{2}$ ', where they might 'violate' a rule in a desperate attempt to arrive at a solution which matches the given answer.

\section{Creating a Taxonomy of Mathematical Errors}

A taxonomy is the "theoretical study of classification, including its bases, principles, procedures and rules" (Simpson, 1961, p.11). It is a way of classifying entities verifiable by observation (Bailey, 1994, p.6). A successful taxonomy will provide classes that are both exhaustive (an appropriate class for each entity) and mutually exclusive (only one suitable class for each entity) (Bailey, 1994, p.3). 
There are a number of major styles of taxonomies used in research, but largely there is a strong relation between how research is conducted and the chosen taxonomy style (Senders and Moray, 1991). For a taxonomy categorising mathematical errors, there are two main styles that immediately seem most viable.

The first style is referred to as a taxonomy of cognitive mechanisms (Senders and Moray, 1991). Here, errors are classified into the stages at which information processing in humans occurs. Often these categorisations come under the following: errors of perception, errors of memory, errors of attention, etc. In other words, the errors of mathematics could be listed under the previously discussed error types. However, as specific errors can be linked to many error types, it becomes significantly harder to distinguish which errors come under which categories.

The second style of taxonomy is a phenomenological taxonomy (Senders and Moray, 1991). In this format, the categories refer almost directly to the errors as they are observed. Typical categories are labelled in the following manner: omissions, substitutions, unnecessary repetitions, etc. This technique of categorisation certainly looks to be the more appropriate choice, especially as there would be fewer discrepancies between the subgroups. Using this method, one would start by first identifying the errors before compiling the headings for each section.

The types of errors made in mathematical assignments can be very different from one another, giving a wide variety of possible mistakes. The majority of previous research was targeted more towards errors made by students studying for GCSE and A-Level, and therefore left many gaps for where undergraduate students may go wrong. Additionally, many areas are sub-discipline specific. For example, the types of errors that may occur whilst tackling an algebraic problem could be different from those which could arise tackling a statistics problem. Some errors are very general, such as communication errors and careless errors.

To be able to identify as many errors that can occur in mathematics as possible, two strategies were used. In the first instance, obvious errors that occur often were first recalled. Secondly, a selection of students' exam scripts from first year courses were analysed to identify other types of errors that had been missed.

The taxonomy that was proposed is given in table 1. Each error is given a code to allow for quick reference to the error when providing feedback to students on their work.

The first group (S) contain errors which are obviously slips of action, a common occurrence in students' mathematical work. Examples include changing the sign of a term from when step to the next, or evaluating $2 \times 3=5$. It could be argued that errors included under 'S3: Incorrect algebraic manipulation' might not be merely due to a careless slip but rather could betray a more fundamental misunderstanding. However, it is not generally possible for a marker to ascertain the reason why a student made a particular error, and since it is to be hoped that undergraduates studying for a mathematics degree are able to perform basic algebraic operations and manipulations competently, these errors are classified under slips of action.

The next group $(U)$ contains errors of understanding - errors which demonstrate a lack of understanding of the mathematics on mathematical concepts being used. These errors go right to the heart of what assessing mathematics is about, and this grouping contains the largest number of errors of all the categories. Examples range from situations where it is clear to the student themselves that there is a gap in their understanding, e.g. where they are unable to finish a solution or arrive at a result which they know to be incorrect, to situations where the student might be oblivious to a fundamental error that they have made, e.g. where they divide by zero or make an argument which is not logically sound. It is of course possible that some of these errors might be merely slips, 
Table 1. Taxonomy of Mathematical Errors.

\begin{tabular}{|c|c|c|}
\hline Code & Error & Examples \\
\hline S1 & Copying error & $\begin{array}{l}\text { Incorrect copying of the question, incorrect copying from one } \\
\text { line to another, mistake copying information into a } \\
\text { graph/diagram }\end{array}$ \\
\hline S2 & $\begin{array}{l}\text { Careless errors on simple } \\
\text { calculations }\end{array}$ & $\begin{array}{l}\text { Errors in addition or multiplication, overlooking negative signs, } \\
\text { cancellation errors }\end{array}$ \\
\hline S3 & $\begin{array}{l}\text { Incorrect algebraic } \\
\text { manipulation }\end{array}$ & $\begin{array}{l}\text { Incorrect roots of equation, incorrect expansion of a bracket, } \\
\text { incorrect handling of powers, incorrect addition/multiplication } \\
\text { of fractions, incorrect partial fractions, incorrect manipulation of } \\
\text { equality }\end{array}$ \\
\hline U1 & $\begin{array}{l}\text { Confusing different } \\
\text { mathematical structures }\end{array}$ & $\begin{array}{l}\text { Stating that a matrix is number, confusing a set with an element } \\
\text { of a set, confusing definite, indefinite and/or improper integrals }\end{array}$ \\
\hline U2 & Incorrect argument & $\begin{array}{l}\text { Claiming an implication which is not true, incorrectly assuming } \\
\text { additivity/commutativity }\end{array}$ \\
\hline U3 & $\begin{array}{l}\text { Lack of consideration of } \\
\text { potential } \\
\text { indeterminate }\end{array}$ & $\begin{array}{l}\text { Division by zero, division by infinity, checking for zero } \\
\text { determinants, checking a function is differentiable, logarithm } \\
\text { not defined on non-positive numbers }\end{array}$ \\
\hline U4 & $\begin{array}{l}\text { Proposed solution is not } \\
\text { viable }\end{array}$ & $\begin{array}{l}\text { Area found is negative, probability found is not between } 0 \text { and } \\
1 \text {, contradictions within solution }\end{array}$ \\
\hline U5 & $\begin{array}{l}\text { Definition/method/theorem } \\
\text { not recalled correctly }\end{array}$ & \\
\hline U6 & Partial solution given & $\begin{array}{l}\text { Correct workings but unfinished solution, did not prove both } \\
\text { implications of a bi-conditional statement }\end{array}$ \\
\hline U7 & Incorrect assumptions & \\
\hline U8 & Misinterpretation of results & \\
\hline CM1 & $\begin{array}{l}\text { Applying an inappropriate } \\
\text { formula/method/theorem }\end{array}$ & $\begin{array}{l}\text { Using irrelevant knowledge, uses the formula/method/theorem } \\
\text { which is not relevant or not valid in the situation }\end{array}$ \\
\hline CM2 & $\begin{array}{l}\text { Correct solution, but a } \\
\text { simpler/quicker approach } \\
\text { could be used }\end{array}$ & Integration could be made simpler by using another method \\
\hline UM1 & $\begin{array}{l}\text { Does not consider all } \\
\text { factors }\end{array}$ & $\begin{array}{l}\text { Not checking all axioms are satisfied, stating conclusions with } \\
\text { insufficient evidence }\end{array}$ \\
\hline UM2 & $\begin{array}{l}\text { Error in use of an } \\
\text { appropriate } \\
\text { definition/method/theorem }\end{array}$ & Algorithm is incorrectly followed, incorrect numbers applied \\
\hline P1 & $\begin{array}{l}\text { Result proved in } \\
\text { specific/restricted cases }\end{array}$ & $\begin{array}{l}\text { Formal proof replaced with specific examples, not proving all } \\
\text { cases }\end{array}$ \\
\hline $\mathrm{P} 2$ & Circular argument & $\begin{array}{l}\text { Using the conclusion of the statement in order to prove the } \\
\text { conclusion }\end{array}$ \\
\hline P3 & $\begin{array}{l}\text { Incorrectly proving } \\
\text { backwards }\end{array}$ & $\begin{array}{l}\text { Incorrectly starting with the conclusion, manipulating it and } \\
\text { arriving at the statement }\end{array}$ \\
\hline C1 & $\begin{array}{l}\text { Undefined variables or } \\
\text { objects }\end{array}$ & \\
\hline $\mathrm{C} 2$ & Notational issues & $\begin{array}{lr}\text { Incorrect use of quantifiers, sets of numbers, summations or } \\
\text { implications, } & \text { omitting } \\
\text { limit/sum/product/differentiation/integration } & \text { symbols, } \\
\text { ambiguously written fractions, no use of brackets } & \end{array}$ \\
\hline C3 & $\begin{array}{l}\text { Graph/diagram presented } \\
\text { poorly }\end{array}$ & $\begin{array}{l}\text { Axes not labelled, important coordinates not labelled, use of } \\
\text { ruler required }\end{array}$ \\
\hline C4 & $\begin{array}{l}\text { Solution difficult to } \\
\mathrm{read} / \text { follow }\end{array}$ & $\begin{array}{l}\text { Bad prose, not communicating in coherent logical flow, } \\
\text { insufficient workings, unclear justifications, unclear what is } \\
\text { being done }\end{array}$ \\
\hline
\end{tabular}


for instance a student may be aware of the danger of dividing by zero but may have simply missed the possibility of this happening in a given situation. However, from experience these kinds of errors are more often made by students who do not appreciate the need for care and precision when making mathematical statements, hence their inclusion as more serious errors of understanding rather than slips of action.

The following two categories (CM, UM) both relate to methods used, with the difference between the two groups being due to the distinction between the two types of rule-based mistakes: misapplication of a good rule and application of a bad rule. The first of these (CM) are errors in choice of method, even though the method itself may have been applied 'correctly', corresponding to the misapplication of a good rule. Here for instance an irrelevant statistical test may have been applied, or a theorem may have been used where the assumptions are not all satisfied. The second group (UM) contains errors in the use of a method, for instance, a student may have missed out an important step in the method.

The next category $(\mathrm{P})$ contains errors specifically related to proof, for example using a circular argument or claiming general results from a few examples. The final category (C) relates to the student's communication of their mathematical solutions, and covers mechanical aspects such as correct use of notation or labelling, as well as more qualitative judgements on clarity of expression or the amount of workings that have been included.

Table 2 shows the alignment of the proposed taxonomy with the model of human errors summarised in figure 2. We see that the taxonomy exhausts the main types of error indicated above. Table 2 shows that each error is typically related quite closely to the other errors within its own category in the taxonomy. Although there is overlap between groups of errors, this overlap has been minimized.

\section{Conclusion}

In this paper we have given a taxonomy of mathematical errors that has been informed by the literature on the different types of human error. This resource has the potential to decrease marking time by enabling tutors to quickly flag up general errors using the short codes for each error (e.g. S1), although it is likely that any feedback provided using the taxonomy would still need to be supplemented with some written comments to provide richer feedback.

If this taxonomy was embedded at programme level and the feedback provided was recorded, then students could be provided with an overall picture summarising the frequency of the types of errors they make, with advice on how to move towards eliminating these errors where possible. It might also be possible to design a resource to help students engage with higher-level reflection upon the types of errors they make, and how they may reduce the probability of these errors occurring. Another possible use of this taxonomy is to inform question setters of the errors they may be likely to see when designing or marking a problem. The notion of informed question design is reminiscent of work by Quinney (2008).

\section{Acknowledgements}

We thank Cardiff University's Centre for Education Innovation for funding the work of the first listed author as part of a Cardiff University Student Education Innovation Project. We are grateful to the two referees for careful reading of our manuscript. 
Table 2. Alignment of the taxonomy with types of error.

\begin{tabular}{|c|c|c|c|c|c|}
\hline Code & 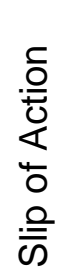 & 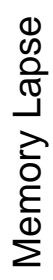 & 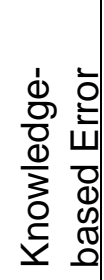 & 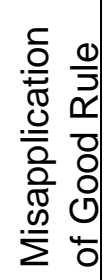 & 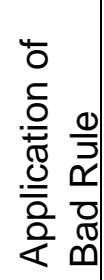 \\
\hline S1 & $X$ & & & & \\
\hline S2 & $X$ & & & & \\
\hline S3 & $X$ & & & & \\
\hline U1 & & $X$ & $X$ & & \\
\hline U2 & & $x$ & $X$ & & \\
\hline U3 & $X$ & $x$ & $X$ & & \\
\hline U4 & $X$ & $x$ & $X$ & & \\
\hline U5 & & $X$ & $x$ & & \\
\hline U6 & & $x$ & $x$ & & \\
\hline U7 & & $x$ & $X$ & & \\
\hline U8 & $x$ & $x$ & $x$ & & \\
\hline $\mathrm{CM} 1$ & & & $x$ & $X$ & \\
\hline $\mathrm{CM} 2$ & & & $x$ & $X$ & \\
\hline UM1 & $x$ & & $x$ & & $x$ \\
\hline UM2 & $X$ & $x$ & $X$ & & $X$ \\
\hline P1 & & $x$ & $X$ & $x$ & \\
\hline P2 & $X$ & & & & $X$ \\
\hline P3 & & & $X$ & & $x$ \\
\hline C1 & $x$ & $x$ & & & \\
\hline $\mathrm{C} 2$ & $x$ & $x$ & $x$ & & \\
\hline C3 & $X$ & $x$ & $X$ & & \\
\hline C4 & $X$ & & $X$ & & \\
\hline
\end{tabular}




\section{References}

Ashlock, R., 2010. Error patterns in computation. 10th ed. Columbus, OH: Pearson.

Bailey, K.D., 1994. Typologies and Taxonomies: An Introduction to Classification Techniques. Thousand Oaks, CA: Sage Publications.

Ben-Zeev, T., 1998. Rational errors and the mathematical mind. Review of General Psychology, 2(4), pp.366-383. https://doi.org/10.1037\%2F1089-2680.2.4.366

Foster, D., 2007. Making meaning in algebra: Examining students' understandings and misconceptions. In: A.H. Schoenfeld, ed. Assessing mathematical proficiency. Cambridge: Cambridge University Press. pp.163-176.

Idris, N., 2011. Error patterns in addition and subtraction for fractions among form two students. Journal of Mathematics Education, 4(2), pp.35-54.

Kieran, C., Booker, G., Filloy, E., Vergnaud, G. and Wheeler, D., 1990. Cognitive processes involved in learning school algebra. In: P. Nesher and J. Kilpatrick, eds. Mathematics and Cognition. Cambridge: Cambridge University Press. Ch. 5.

MacGregor, M. and Stacey, K., 1997. Students understanding of algebraic notation. Educational Studies in Mathematics, 33(1), pp.1-19. https://doi.org/10.1023/A:1002970913563

Matz, M., 1982. Towards a process model for school algebra error. In: D. Sleeman and J.S. Brown, eds. 1982. Intelligent tutoring systems. New York: Academic Press. pp.25-50.

Quinney, D., 2008. So just what is conceptual understanding of mathematics? MSOR Connections, 8(3), pp.2-7.

Radatz, H., 1979. Error analysis in mathematics education. Journal for Research in Mathematics Education, 10(3), pp.163-172. https://doi.org/10.2307/748804

Radatz, H., 1980. Students' errors in the mathematical learning process: a survey. For the Learning of Mathematics, 1(1), pp.16-20.

Rasmussen, J., 1986. Information Processing and Human-Machine Interaction: An Approach to Cognitive Engineering. New York: Elsevier Science.

Reason, J., 1990. Human Error. Cambridge: Cambridge University Press.

Senders, J. and Moray, N., 1991. Human Error: Causes, Prediction and Reduction. Hillsdale, NJ: Lawrence Erlbaum Associates.

Simpson, G.G., 1961. Principles of Animal Taxonomy. New York: Columbia University Press. 